\title{
MAGNETIC PROPERTIES OF FERROFLUID EMULSIONS: THE EFFECT OF DROPLET ELONGATION
}

\author{
A.O. Ivanov, O.B. Kuznetsova, I.M. Subbotin \\ Institute of Mathematics and Computer Sciences, Ural Federal University \\ 51 Lenin Ave., 620000 Ekaterinburg, Russia
}

\begin{abstract}
The paper is concerned with a theoretical explanation of the experimentally observed effect of non-monotonic field dependence of the effective magnetic permeability of ferrofluid emulsion. In a weak magnetic field, the growth of the induced droplet magnetic moment is faster than the linear one due to the droplet elongation accompanied by the reduction of the demagnetizing field. Thus, the emulsion magnetic permeability increases in weak magnetic fields. Further strengthening of the external magnetic field cannot lead to a significant decrease of the demagnetizing field, as the droplets are already highly elongated. On the other hand, the magnetic susceptibility of the ferrofluid reduces with the field strength. Hence, the effective magnetic permeability of the ferrofluid emulsion starts decreasing. The developed theoretical model describes well the experimental observations.
\end{abstract}

1. Introduction. Ferrofluid emulsions are colloidal suspensions of micronsized droplets, filled with a ferrofluid, in an immiscible neutral liquid. An applied magnetic field induces a magnetic dipole moment in each droplet, therefore, the ferrofluid becomes magnetized. So the induced droplet magnetic moment is dependent on the droplet volume and on the magnetization behaviour of the bulk ferrofluid. Recently, an unexpected experimental field dependence of magnetic permeability has been reported in [1] for ferrofluid emulsions (see Fig. 2 hereinafter, experimental dots), produced by dispersing a kerosene-based ferrofluid in an immiscible aviation oil. The weak-field permeability demonstrates the rapid growth, and the further field strengthening leads to a long-tail decrease of the magnetic permeability. The effect could be observed only for ferrofluid emulsions characterized by rather weak values of interfacial tension $\left(\sim 10^{-6} \mathrm{~N} / \mathrm{m}\right)$. The maximum value of the effective emulsion permeability increases with the volume concentration of the droplets. During the measurements, the droplet coalescence was not experimentally observed in [1]. The suspension may be inverted to get an "inversed" ferrofluid emulsion, representing a suspension of liquid droplets in a carrier ferrofluid. The non-monotonic field dependence of magnetic permeability was also observed for the inverted ferrofluid emulsion [1].

Here we suggest a theoretical explanation of this effect based on the following physical idea. The weak-field growth of magnetic permeability is caused by the droplet elongation and by the resulting reduction of the demagnetizing field. Due to the latter, the induced droplet magnetic moments grow more rapidly with the field strength than linearly. Thus, the effective emulsion magnetic permeability increases. In stronger magnetic fields, the droplets are highly elongated and further reduction of the demagnetizing field is not important. At the same time, the ferrofluid magnetic susceptibility decreases with the field. So the droplets become less sensitive to further strengthening of the magnetic field, and the emulsion magnetic permeability decreased. To prove this explanation, we combine the known theoretical results on the static magnetization behaviour of ferrofluids, the solution of magnetostatic problem for the magnetic field geometry inside and outside 
the droplet, and the minimization approach of the droplet energy to obtain the degree of droplet elongation. Unlike the previously published theory [2] based on the assumption of individual non-interacting droplets, we prove here the applicability of the effective "mean-field" method account for the inter-droplet magnetic influence proposed heuristically in [3].

2. Model of interacting droplets. The main approximations of our model are the following. All ferrofluid droplets are considered to be of the equal volume $V_{\mathrm{d}}$. The shape of the stretched droplets is modeled by the elongated ellipsoid of revolution; in this case, the internal magnetic field inside the droplet is uniform. The magnetic permeability of the carrier liquid (oil) is considered to be equal to unity. To describe the magnetic parameters of the ferrofluid, we use the firstorder modified mean-field model [4], which has been proved as giving excellent agreement with experiments and computer simulations both for moderately concentrated ferrofluids $[5-7]$ and for ferrogels $[8,9]$. In the framework of this model, the ferrofluid magnetization $M_{\mathrm{d}}$ is given by

$$
M_{\mathrm{d}}(H)=M_{\mathrm{L}}\left[H+\frac{4 \pi}{3} M_{\mathrm{L}}(H)\right], \quad M_{\mathrm{L}}(H)=M_{\mathrm{s}} L\left(m H / k_{\mathrm{B}} T\right) .
$$

Here $H$ is the magnetic field strength, $L(x)$ and $M_{\mathbf{L}}(H)$ are, respectively, the Langevin function and the Langevin magnetization; $M_{\mathrm{s}}$ has a meaning of the ferrofluid saturation magnetization, $m$ stands for the ferroparticle magnetic moment, and $k_{\mathrm{B}} T$ is the thermal energy. Simple fitting of the experimental data on the magnetic permeability of the ferrofluid used in the emulsions under study [1] gives the important effective parameters: the ferrofluid saturation magnetization [3] $M_{\mathrm{s}}=20.3 \mathrm{kA} / \mathrm{m}$, and the ferrofluid initial magnetic susceptibility $4 \pi \chi_{\mathrm{f}}=5.4$.

The emulsion magnetic permeability $\mu_{\mathrm{e}}$ could be defined as [2]

$$
\mu_{\mathrm{e}}=1+4 \pi \varphi M_{\mathrm{d}}\left(H_{\mathrm{d}}\right) / H_{0}
$$

where $\varphi$ stands for the droplet volume fraction, and the droplet magnetization $M_{\mathrm{d}}$ is the function of the strength of the internal magnetic field $H_{\mathrm{d}}$ inside the droplet. This internal field is determined by the solution of the magnetostatic problem, defining the magnetic field geometry inside and outside the magnetizing droplet exposed to a uniform external static field $H_{0}$. Each droplet is magnetized due to the action both of the external field $H_{0}$ and of the collective magnetic field produced by other droplets. Since this problem could not be solved for an ensemble of randomly positioned elongated droplets, we use here the iteration procedure. As the first step, we choose one droplet and solve the magnetostatic problem completely neglecting other droplets. The solution gives the well-known relation, connecting the first-step internal field $H_{\mathrm{d}}^{(1)}$, the droplet magnetization $M_{\mathrm{d}}$, the external field $H_{0}$, and the ellipsoid eccentricity $e$ via the demagnetization factor $n_{z}(e)$

$$
H_{0}=H_{\mathrm{d}}^{(1)}+4 \pi n_{z}(e) M_{\mathrm{d}}\left(H_{\mathrm{d}}^{(1)}\right), \quad n_{z}(e)=\frac{1-e^{2}}{2 e^{3}}\left(\ln \frac{1+e}{1-e}-2 e\right) .
$$

Since the effect is pronounced mainly in the weak field region, the droplets are quasi-spherical here, and they produce a magnetic field approximately of dipole type. As the second step, we summarize the dipole-type magnetic fields produced by all randomly positioned droplets in the region occupied by the first one. The important point is that this collective magnetic field appears to be uniform [10] 
Magnetic properties of ferrofluid emulsions: the effect of droplet elongation

and is equal to $4 \pi n_{z}(e) \varphi M_{\mathrm{d}}\left(H_{\mathrm{d}}^{(1)}\right)$. So, we add this collective field to the external one and use solution (3) to obtain an internal field $H_{\mathrm{d}}^{(2)}$ at the second step:

$$
H_{0}+4 \pi n_{z}(e) \varphi M_{\mathrm{d}}\left(H_{\mathrm{d}}^{(1)}\right)=H_{\mathrm{d}}^{(2)}+4 \pi n_{z}(e) M_{\mathrm{d}}\left(H_{\mathrm{d}}^{(2)}\right) .
$$

Repeating the steps, we get the iteration relation

$$
H_{0}+4 \pi n_{z}(e) \varphi M_{\mathrm{d}}\left(H_{\mathrm{d}}^{(i)}\right)=H_{\mathrm{d}}^{(i+1)}+4 \pi n_{z}(e) M_{\mathrm{d}}\left(H_{\mathrm{d}}^{(i+1)}\right),
$$

which is converging to the nonlinear equation for determining the internal magnetic field $H_{\mathrm{d}}$ at each given external field strength $H_{0}$, droplet concentration $\varphi$ and eccentricity $e$ :

$$
H_{0}=H_{\mathrm{d}}+4 \pi n_{z}(e)(1-\varphi) M_{\mathrm{d}}\left(H_{\mathrm{d}}\right) .
$$

In the weak field limit, when the linear magnetization law $M_{\mathrm{d}}=\chi_{\mathrm{f}} H_{\mathrm{d}}$ holds true, Eqs. (2), (5) could be easily solved, and we get the initial (zero-field) value for the magnetic permeability

$$
\mu_{\mathrm{e}}=1+\frac{4 \pi \chi_{\mathrm{f}} \varphi}{1+4 \pi \chi_{\mathrm{f}} n_{z}(e)(1-\varphi)}
$$

which is valid for suspensions of ellipsoidal or anisotropic particles, containing point dipoles at the center. For the case of ferrofluid emulsions in the weak field limit, all droplets are spherical, and the demagnetization factor is equal to $1 / 3$. So expression (6) turns out to the known Maxwell-Wagner formula

$$
\mu_{\mathrm{e}}=1+\frac{4 \pi \chi_{\mathrm{f}} \varphi}{1+4 \pi \chi_{\mathrm{f}}(1-\varphi) / 3}
$$

which does not contain any adjustable parameter instead of the known initial magnetic susceptibility $\chi_{\mathrm{f}}$ of the ferrofluid inside the droplets. This expression demonstrates the nonlinear concentration dependence of the initial magnetic permeability of the emulsion. The comparison with the experimental data from [1] is presented in Fig. 1. The very accurate quantitative agreement substantiates the necessity of taking into account the inter-droplet interaction.

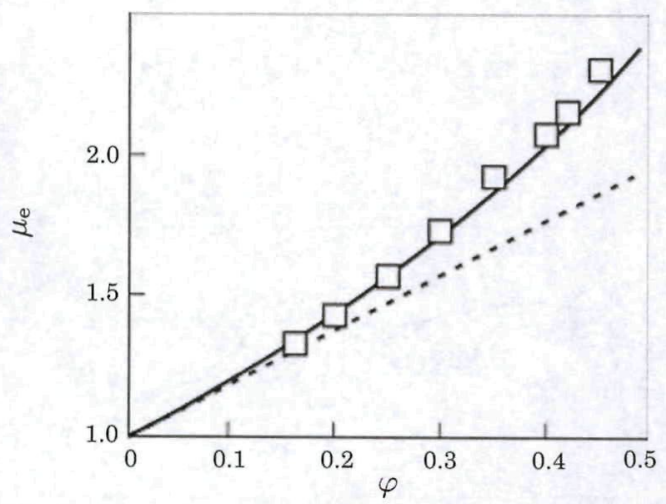

Fig. 1. Concentration dependence of the initial magnetic permeability of the ferrofluid emulsion. The experimental data [1] are indicated with boxes. The dotted line describes the linear concentration dependence predicted by the model of non-interacting droplets [2]. The nonlinear behaviour obtained on the basis of the effectively interacting droplet model Eq. (7) is given by the solid line. 
3. Free energy minimization. To determine the degree of droplet elongation at each given external field, we use the free energy minimization approach. We assume the droplet free energy as a sum of surface energy and magnetic energy

$$
\begin{aligned}
F=\sigma S_{\mathrm{d}}-V_{\mathrm{d}} \int_{0}^{H_{0}} M_{\mathrm{d}}\left(H_{\mathrm{d}}\right) \mathrm{d} H_{0} & =\sigma S_{\mathrm{d}}-V_{\mathrm{d}} \int_{0}^{H_{\mathrm{d}}} M_{\mathrm{d}}\left(H_{\mathrm{d}}\right) \mathrm{d} H_{\mathrm{d}}-4 \pi n_{z}(e) V_{\mathrm{d}} \frac{M_{\mathrm{d}}^{2}}{2}, \\
S_{\mathrm{d}}(e) & =2 \pi\left(\frac{3 V_{\mathrm{d}}}{4 \pi}\right)^{2 / 3}\left(1-e^{2}\right)^{1 / 3}\left(1+\frac{\arcsin e}{e \sqrt{1-e^{2}}}\right),
\end{aligned}
$$

where $\sigma$ is the interfacial tension; and the ellipsoid surface area $S_{\mathrm{d}}$ is expressed in terms of the droplet volume $V_{\mathrm{d}}$ and eccentricity e. Calculating the minimum yields the following expression

$$
\frac{2 \pi V_{\mathrm{d}} M_{\mathrm{d}}\left(H_{\mathrm{d}}\right)^{2}}{\sigma}=-\frac{\mathrm{d} S_{\mathrm{d}}(e) / \mathrm{d} e}{\mathrm{~d} n_{z}(e) / \mathrm{d} e} .
$$

It is worth noting that the shape of a ferrofluid droplet exposed to an external magnetic field could be also studied within a pressure approach, since the driven force of the droplet elongation is the magnetic pressure. This method was described in [11], and the obtained relation for the case of arbitrary magnetic field is coincident with that of Eq. (9).

The final set of equations, at each given external field strength $H_{0}$ determining the internal field $H_{\mathrm{d}}$, the droplet magnetization $M_{\mathrm{d}}$, and the droplet eccentricity e, includes Eqs. (1), (5) and (9). The ferrofluid emulsion magnetic permeability is calculated based on Eq. (2). The obtained theoretical results are presented in Fig. 2 for the ferrofluid emulsions with the droplet volume fractions $\varphi=0.2$ and 0.35 . The droplet radius is put to $5 \mu \mathrm{m}$ and the interfacial tension is chosen to be $5 \times 10^{-7} \mathrm{~N} / \mathrm{m}$. We get the accurate quantitative agreement for the weak field growth, but the theoretical curves are shifted to the right in stronger fields. Several reasons can be suggested for the explanation of these deviations. Among them is

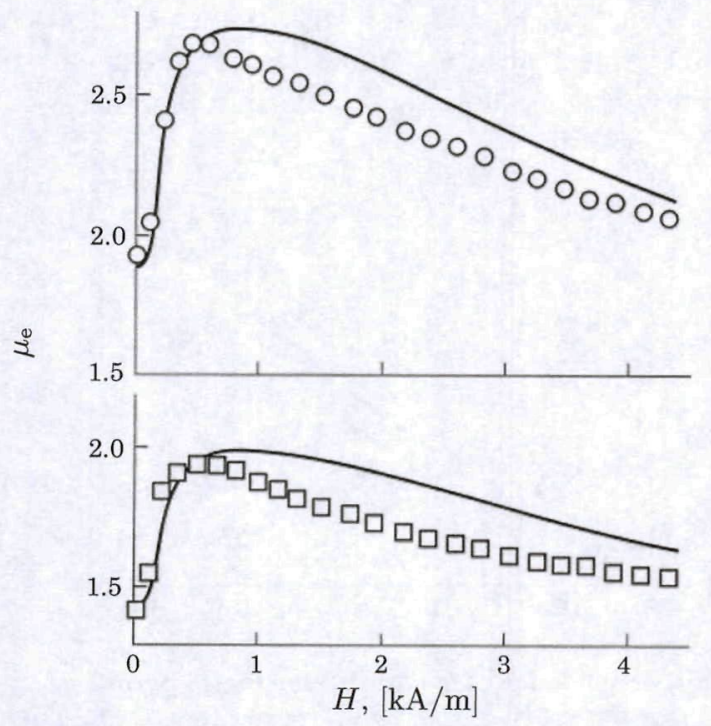

Fig. 2. Magnetic permeability $\mu_{\mathrm{e}}$ of the ferrofluid emulsion as a function of the external field $H$ strength. Experimental data [1]: $\square-\varphi=0.2, \circ-\varphi=0.35$. Curves show the theoretical results. 
an oversimplified approximation of the droplet shape with the elongated ellipsoid of revolution, or the high elongation of the droplets may result in their breaks. Also, the magnetic nanoparticles may diffuse from the droplets into the bulk carrier liquid due to the magnetophoresis induced by the field gradients in the local vicinity of the droplets. This uncontrolled flux will lead to the decrease of the ferrofluid saturation magnetization, which we consider as fixed. All these effects may slightly affect the decreasing behaviour of the emulsion magnetic permeability in stronger magnetic fields. However, the major contribution to this decrease is made by the weakening of the magnetic response of the ferrofluid inside the droplets. The presented model mainly accounts for this effect, and it gives a qualitatively and quantitatively correct theoretical explanation of the experimentally observed non-monotonic field behaviour of the magnetic permeability of ferrofluid emulsion.

\section{Conclusions.}

- Ferrofluid emulsions are the interesting objects of magnetic soft matter, containing structural units (ferrofluid droplets) of variable shape.

- The presented simple model describes correctly the non-monotonic field dependence of the ferrofluid emulsion magnetic permeability.

- Weak field growth of the magnetic permeability is caused by the droplet elongation and by the resulting reduction of the demagnetizing field.

- The strong field decrease of the magnetic permeability is explained by the decay of the ferrofluid magnetic susceptibility under termination of further droplet elongation.

5. Acknowledgements. This research was carried out under the financial support from the Russian Foundation for Basic Research and the Government of Sverdlovsk Region, grant No. 13-01-96032_r_ural, and was partly supported by the Ministry of Education and Science of the Russian Federation, project No.2.609.2011.

\section{REFERENCES}

[1] Yu.I. Dikanskit, A.R. Zakinyan, and N.Yu. Konstantinova. On the permeability of a magnetodielectric emulsion. Technical Physics, vol. 53 (2008), no. 1, pp. 19-23.

[2] A.O. Ivanov, O.B. Kuznetsova, And I.M. Subbotin. Magnetic properties of ferrofluid emulsions: model of non-interacting droplets. Magnetohydrodynamics, vol. 47 (2011), no. 2, pp. 129-134.

[3] A.O. Ivanov, and O.B. Kuznetsova. Nonmonotonic field-dependent magnetic permeability of a paramagnetic ferrofluid emulsion. Phys. Rev. E, vol. 85 (2011), no. 4, p. 041405-01-06.

[4] A.O. Ivanov, and O.B. Kuznetsova. Magnetogranulometric analysis of ferrocolloids: modified mean-field model of the second order. Colloid J., vol. 68 (2006), no. 4 , pp. 430-440.

[5] A.O. Ivanov et al. Magnetic properties of polydisperse ferrofluids: a critical comparison between experiment, theory, and computer simulation. Phys. Rev. $E$, vol. 75 (2007), no. 6, p. 061405-01-12.

[6] V.M. Polunin et al. On the estimation of physical parameters of magnetic nanoparticles in magnetic fluid. Magnetohydrodynamics, vol. 46 (2010), no. 1, pp. 31-40. 
[7] A.M. Storozhenko et al. Interaction of physical fields under the acoustomagnetic effect in magnetic fluids. Magnetohydrodynamics, vol. 47 (2011), no. 4 , pp. $345-358$.

[8] D.S. Wood, AND P.J. CAMP. Modeling the properties of ferrogels in uniform magnetic fields. Phys. Rev. E, vol. 83 (2011), no. 1, pp. 011402-01-09.

[9] Рн.J. CAmp. The effects of magnetic fields on the properties of ferrofluids and ferrogels. Magnetohydrodynamics, vol. 47 (2011), no. 2, pp. 123-128.

[10] C.V. HeEr. Statistical Mechanics, Kinetic Theory, and Stochastic Processes (Academic Press, New-York and London, 1972).

[11] E. Blums, A. Cebers, And M.M. Maiorov. Magnetic Fluids (W. de Gruyter, Berlin, 1997).

Received 06.05.2013 
Copyright of Magnetohydrodynamics (0024-998X) is the property of University of Latvia, Institute of Physics and its content may not be copied or emailed to multiple sites or posted to a listserv without the copyright holder's express written permission. However, users may print, download, or email articles for individual use. 